\title{
Inequalities involving Hadamard products of centrosymmetric matrices
}

\author{
Mustafa Ozel ${ }^{1}$ and Dilek Kayaalp ${ }^{2}$ \\ ${ }^{1}$ Dokuz Eylul University Faculty of Engineering, Department of Geophysics Engineering, Tinaztepe Campus, Buca, Izmir, Turkey \\ ${ }^{2}$ Dokuz Eylul University, The Graduate School of Natural and Applied Sciences, Tinaztepe Campus, Buca, Izmir, Turkey
}

Received: 12 March 2018, Accepted: 14 December 2018

Published online: 23 December 2018.

\begin{abstract}
Some new results on Kronecker and Hadamard products of centrosymmetric matrices and their equivalence forms are discussed, respectively. In addition, we derive an upper bound for the spectral radius of Hadamard product of two centrosymmetric matrices $A$ and $B$ with respect to $p$-norm of blocks of $A \circ B$, for $p \geq 2$.
\end{abstract}

Keywords: Spectral radius, Hadamard product, Kronecker product, p-norm, centrosymmetric matrix.

\section{Introduction and Preliminaries}

A centrosymmetric matrix being symmetric about its center has wide range of applications in antenna array, quantum physics, mechanical and electrical systems, pattern recognition, communication theory, speech analysis, digital filters and linear prediction. The structure of centrosymmetric matrices provides computationally efficient results in complex algorithms. Many applications such as pattern recognition feature selection, a uniform linear antenna array, vibration in structures and quantum mechanical oscillator benefit from this inherent structure of centrosymmetric matrices $[2,3,4]$.

Let $n$ be a positive integer. Then, $\mathbb{R}^{n \times n}$ denote the set of all real matrices throughout the paper. Let $A=\left[a_{i j}\right]$ and $B=\left[b_{i j}\right]$ be two real $n \times n$ matrices. Then, we have $A \geq B(>B)$ if $a_{i j} \geq b_{i j}\left(>b_{i j}\right)$ for all $1 \leq i \leq n, 1 \leq j \leq n$. If $A \geq 0$ (>0), we say $A$ is nonnegative (positive) matrix, respectively. The spectral radius of $A$ is denoted by $\rho(A)$. If $A$ is a nonnegative matrix, the Perron-Frobenius theorem guarantees that $\rho(A) \in \sigma(A)$, where $\sigma(A)$ denotes the spectrum of $A$. The Hadamard product of $A, B \in \mathbb{R}^{n \times n}$ is denoted by $A \circ B=\left[a_{i j} b_{i j}\right] \in \mathbb{R}^{n \times n}$. The Kronecker product of two matrices $A=\left[a_{i j}\right] \in \mathbb{R}^{m \times n}$ and $B=\left[b_{k l}\right] \in \mathbb{R}^{p \times q}$ is the matrix $A \otimes B=\left[a_{i j} B\right]$ of order $m p \times n q$. The identity matrix in $\mathbb{R}^{n}$ is denoted by $I_{n}$. As usual, $A^{*}=(\bar{A})^{T}$ denotes the conjugate transpose of matrix $A$. A Hermitian matrix $A$ is called positive semidefinite if $x^{*} A x \geq 0$ for all $x \in \mathbb{C}^{n}$, and is called positive definite if $x^{*} A x>0$ for all nonzero $x \in \mathbb{C}^{n}[1,2,5,6,10]$. The $p$-norm of an $m \times n$ matrix $A$ is defined as

$$
\|A\|_{p}=\left(\operatorname{tr}\left(A^{*} A\right)^{p / 2}\right)^{1 / p}
$$

Definition 1. $A=\left[a_{i j}\right]_{n \times n}$ is a centrosymmetric matrix, if $a_{i j}=a_{n-i+1, n-j+1}$ where $1 \leq i \leq n, 1 \leq j \leq n$, or $J_{n} A J_{n}=A$ where $J_{n}$ is the flip matrix with ones on the secondary diagonal and zeroes elsewhere [5, 8, 9,11]. 
Lemma 1.Let $A=\left[a_{i j}\right]_{n x n}$ be a centrosymmetric matrix in one of the following forms

$$
A=\left[\begin{array}{cc}
A_{1} & J_{m} A_{2} J_{m} \\
A_{2} & J_{m} A_{1} J_{m}
\end{array}\right] \quad \text { or } \quad A=\left[\begin{array}{ccc}
A_{1} & J_{m} a_{2} & J_{m} A_{2} J_{m} \\
a_{1}^{T} & \alpha & a_{1}^{T} J_{m} \\
A_{2} & a_{2} & J_{m} A_{1} J_{m}
\end{array}\right]
$$

Then, for $B, C \in \mathbb{R}^{m \times m}, a_{1}, a_{2} \in \mathbb{R}^{m \times 1}, \alpha$ is a scalar, we have either

$$
Q_{1}^{T} A Q_{1}=\left[\begin{array}{cc}
A_{1}-J_{m} A_{2} & 0 \\
0 & A_{1}+J_{m} A_{2}
\end{array}\right]
$$

or

$$
Q_{2}^{T} A Q_{2}=\left[\begin{array}{ccc}
A_{1}-J_{m} A_{2} & 0 & 0 \\
0 & \alpha & \sqrt{2} a_{1}^{T} \\
0 & \sqrt{2} J_{m} a_{2} & A_{1}+J_{m} A_{2}
\end{array}\right]
$$

where $Q_{1}=\frac{1}{\sqrt{2}}\left[\begin{array}{cc}I_{m} & I_{m} \\ -J_{m} & J_{m}\end{array}\right]$ and $Q_{2}=\frac{1}{\sqrt{2}}\left[\begin{array}{ccc}I_{m} & 0 & I_{m} \\ 0 & \sqrt{2} & 0 \\ -J_{m} & 0 & J_{m}\end{array}\right]$.

We have the following facts on the spectral radius of a matrix and the properties of Hadamard and Kronecker products [1, $2,5,6,7,8,10]$.

Lemma 2. Let $A, B \in \mathbb{R}^{n \times n}$. Then we have

(1) $\rho(A) \leq\|A\|$ for any norm.

(2) If $|A| \leq B$, then $\rho(A) \leq \rho(|A|) \leq \rho(B)$ where $|A|=\left[\left|a_{i j}\right|\right]_{n \times n}$ and $\rho(A)$ is the spectral radius of $A$.

We use some properties of Hadamard and Kronecker products throughout. One we use is the following property of Kronecker product:

$$
(A \otimes B)(C \otimes D)=A C \otimes B D
$$

for $A_{p \times q}, B_{s \times t}, C_{q \times u}$ and $D_{t \times v}$. The other one is the relation between Kronecker and Hadamard products given in the following lemma.

Lemma 3. Let $A$ and $B$ be $n \times n$ matrices. Then there exists an $n^{2} \times n$ selection matrix $\mathbb{J}$ such that $\mathbb{J}^{T} \mathbb{J}=I$ and

$$
A \circ B=\mathbb{J}^{T}(A \otimes B) \mathbb{J}
$$

where $\mathbb{J}^{T}$ is an $n \times n^{2}$ matrix $\left[E_{11}, E_{22}, \ldots, E_{n n}\right]$ and $E_{i i}$ is the $n \times n$ matrix of zeros except for a one in the $(i, i)$ th position.

\section{Main results}

In this section, we mainly discuss some results on Hadamard and Kronecker products of two centrosymmetric matrices. In [3], Chen, Wang, and Zhong mentioned the following result on centrosymmetric matrices. Here, we give a proof of the result.

Proposition 1. Let $A=\left[a_{i j}\right]_{n \times n}$ and $B=\left[b_{i j}\right]_{n \times n}$ be two centrosymmetric matrices. Then $A \otimes B$ is a centrosymmetric matrix. 
Proof. Using the definition of Kronecker product for $A$ and $B$, we have

$$
A \otimes B=\left[\begin{array}{c}
A_{1} \otimes B\left(J_{m} A_{2} J_{m}\right) \otimes B \\
A_{2} \otimes B\left(J_{m} A_{1} J_{m}\right) \otimes B
\end{array}\right] \text { for } n=2 m
$$

and

$$
A \otimes B=\left[\begin{array}{ccc}
A_{1} \otimes B & J_{m} a_{2} \otimes B & \left(J_{m} A_{2} J_{m}\right) \otimes B \\
a_{1}^{T} \otimes B & \alpha \otimes B & a_{1}^{T} J_{m} \otimes B \\
A_{2} \otimes B & a_{2} \otimes B & \left(J_{m} A_{1} J_{m}\right) \otimes B
\end{array}\right] \text { for } n=2 m+1
$$

It is enough to show that

$$
J_{m n}\left(A_{1} \otimes B\right) J_{m n}=\left(J_{m} A_{1} J_{m}\right) \otimes B \text { and } J_{n}\left(a_{1}^{T} \otimes B\right) J_{m n}=\left(a_{1}^{T} J_{m}\right) \otimes B
$$

Indeed since $B$ is centrosymmetric and $J_{m n}=J_{m} \otimes J_{n}$ we have

$$
J_{m n}\left(A_{1} \otimes B\right) J_{m n}=\left(J_{m} \otimes J_{n}\right)\left(A_{1} \otimes B\right)\left(J_{m} \otimes J_{n}\right)=\left(J_{m} A_{1} J_{m}\right) \otimes B
$$

and

$$
J_{n}\left(a_{1}^{T} \otimes B\right) J_{m n}=\left(1 \otimes J_{n}\right)\left(a_{1}^{T} \otimes B\right)\left(J_{m} \otimes J_{n}\right)=a_{1}^{T} J_{m} \otimes B .
$$

Therefore, $A \otimes B$ is a centrosymmetric matrix.

It was given by Zhao, Li and Gong in [11] that Hadamard product of two centrosymmetric matrices is centrosymmetric. We give an alternative proof for this statement. For the proof, we need the following relation between flip and selection matrices.

Proposition 2. Let $J_{m}$ and $\mathbb{J}_{m^{2} \times m}$ be the flip and the selection matrices, respectively. Then

(1) $\mathbb{J}_{m \times m^{2}}^{T} J_{m^{2}}=J_{m} \mathbb{J}_{m \times m^{2}}^{T}$

(2) $J_{m^{2}} \mathbb{J}_{m^{2} \times m}=\mathbb{J}_{m^{2} \times m} J_{m}$.

Proof. If $J_{m}$ is an $m \times m$ flip matrix and $\mathbb{J}_{m \times m^{2}}^{T}$ is an $m \times m^{2}$ selection matrix,

$$
\begin{aligned}
\mathbb{J}_{m \times m^{2}}^{T} J_{m^{2}} & =\left[E_{11}, E_{22}, \ldots, E_{m m}\right] J_{m^{2}} \\
& =\left[E_{m m} J_{m}, E_{m-1, m-1} J_{m}, \ldots, E_{22} J_{m}, E_{11} J_{m}\right] \\
& =\left[J_{m} E_{11}, J_{m} E_{22}, \ldots, J_{m} E_{m-1, m-1}, J_{m} E_{m m}\right] \\
& =J_{m}\left[E_{11}, E_{22}, \ldots, E_{m m}\right] \\
& =J_{m} \mathbb{J}_{m \times m^{2}}^{T} .
\end{aligned}
$$

Thus, the proof of part (1) is completed. The second part clearly follows from (1) since $J_{m}^{T}=J_{m}$.

We define a centrosymmetric matrix $B$ in one of the following two forms

$$
B=\left[\begin{array}{cc}
B_{1} & J_{m} B_{2} J_{m} \\
B_{2} & J_{m} B_{1} J_{m}
\end{array}\right] \quad \text { or } \quad B=\left[\begin{array}{ccc}
B_{1} & J_{m} b_{2} & J_{m} B_{2} J_{m} \\
b_{1}^{T} & \beta & b_{1}^{T} J_{m} \\
B_{2} & b_{2} & J_{m} B_{1} J_{m}
\end{array}\right]
$$

for $n=2 m$ or $n=2 m+1$, respectively.

Theorem 1. Let $A=\left[a_{i j}\right]_{n x n}$ and $B=\left[b_{i j}\right]_{n x n}$ be two centrosymmetric matrices as in (1) and (6), then $A \circ B$ is a centrosymmetric matrix. 
Proof.Using the definition of Hadamard product of $A$ and $B$ for $n=2 m$ and $n=2 m+1$, we have

$$
A \circ B=\left[\begin{array}{ll}
A_{1} \circ B_{1} & \left(J_{m} A_{2} J_{m}\right) \circ\left(J_{m} B_{2} J_{m}\right) \\
A_{2} \circ B_{2} & \left(J_{m} A_{1} J_{m}\right) \circ\left(J_{m} B_{1} J_{m}\right)
\end{array}\right]
$$

and

$$
A \circ B=\left[\begin{array}{ccc}
A_{1} \circ B_{1} & J_{m} a_{2} \circ J_{m} b_{2} & \left(J_{m} A_{2} J_{m}\right) \circ\left(J_{m} B_{2} J_{m}\right) \\
a_{1}^{T} \circ b_{1}^{T} & \alpha \beta & a_{1}^{T} J_{m} \circ b_{1}^{T} J_{m} \\
A_{2} \circ B_{2} & a_{2} \circ b_{2} & \left(J_{m} A_{1} J_{m}\right) \circ\left(J_{m} B_{1} J_{m}\right)
\end{array}\right]
$$

To prove $A \circ B$ is a centrosymmetric matrix, we need to show that

$$
\left(J_{m} A_{1} J_{m}\right) \circ\left(J_{m} B_{1} J_{m}\right)=J_{m}\left(A_{1} \circ B_{1}\right) J_{m} \text { and } J_{1}\left(a_{1}^{T} \circ b_{1}^{T}\right) J_{m}=a_{1}^{T} J_{m} \circ b_{1}^{T} J_{m}
$$

Note that the second equation clearly follows. For the first one, by Proposition 2 and Lemma 3, we have

$$
\begin{aligned}
\left(J_{m} A_{1} J_{m}\right) \circ\left(J_{m} B_{1} J_{m}\right) & =\mathbb{J}_{m \times m^{2}}^{T}\left[\left(J_{m} A_{1} J_{m}\right) \otimes\left(J_{m} B_{1} J_{m}\right)\right] \mathbb{J}_{m^{2} \times m} \\
& =\mathbb{J}_{m \times m^{2}}^{T}\left[\left(J_{m} \otimes J_{m}\right)\left(A_{1} \otimes B_{1}\right)\left(J_{m} \otimes J_{m}\right) \mathbb{J}_{m^{2} \times m}\right. \\
& =\mathbb{J}_{m \times m^{2}}^{T}\left(J_{m^{2}}\left(A_{1} \otimes B_{1}\right) J_{m^{2}}\right) \mathbb{J}_{m^{2} \times m} \\
& =\mathbb{J}_{m \times m^{2}}^{T} J_{m^{2}}\left(A_{1} \otimes B_{1}\right) J_{m^{2}} \mathbb{J}_{m^{2} \times m} \\
& =J_{m} \mathbb{J}_{m \times m^{2}}^{T}\left(A_{1} \otimes B_{1}\right) \mathbb{J}_{m^{2} \times m} J_{m} \\
& =J_{m}\left(\mathbb{J}_{m \times m^{2}}^{T}\left(A_{1} \otimes B_{1}\right) \mathbb{J}_{m^{2} \times m}\right) J_{m} \\
& =J_{m}\left(A_{1} \circ B_{1}\right) J_{m} .
\end{aligned}
$$

We note that by Lemma $1, A \circ B$ has one of the following two forms

$$
\begin{aligned}
& Q_{1}^{T}(A \circ B) Q_{1}=\left[\begin{array}{cc}
P-J_{m} S & 0 \\
0 & P+J_{m} S
\end{array}\right] \text { for } n=2 m \text { or } \\
& Q_{2}^{T}(A \circ B) Q_{2}=\left[\begin{array}{cc}
P-J_{m} S & 0 \\
0 & K
\end{array}\right] \text { for } n=2 m+1,
\end{aligned}
$$

where $P=A_{1} \circ B_{1}, S=A_{2} \circ B_{2}$, and $K=\left[\begin{array}{cc}\alpha \beta & \sqrt{2}\left(a_{1}^{T} \circ b_{1}^{T}\right) \\ \sqrt{2} J_{m}\left(a_{2} \circ b_{2}\right) & P+J_{m} S\end{array}\right]$.

Li, Zhao, Dai and Su in [8] had proved that for a nonnegative centrosymmetric matrix it is possible to find the spectral radius of a matrix in terms of its blocks. In fact, if $A$ and $B$ are centrosymmetric matrices, then we have

$$
\begin{array}{ll}
\rho(A \circ B)=\rho\left\{A_{1} \circ B_{1}+J_{m}\left(A_{2} \circ B_{2}\right)\right\} \quad \text { for } n=2 m & \text { and } \\
\rho(A \circ B)=\max \left\{\rho(K), \rho\left(A_{1} \circ B_{1}-J_{m}\left(A_{2} \circ B_{2}\right)\right)\right\} & \text { for } n=2 m+1 .
\end{array}
$$

Now we give an upper bound for the spectral radius of Hadamard product of two nonnegative centrosymmetric matrices. 
Proposition 3.Let $A=\left[a_{i j}\right]_{n \times n}$ and $B=\left[b_{i j}\right]_{n \times n}$ be two nonnegative centrosymmetric matrices, then

$$
\begin{aligned}
& \rho(A \circ B) \leq \rho\left(A_{1}^{T} B_{1}\right)+m^{1 / p} \rho\left(A_{2}^{T} B_{2}\right) \text { for } n=2 m \quad \text { and } \\
& \quad \rho(A \circ B) \leq \max \left\{\|K\|_{p},\left\|A_{1} \circ B_{1}-J_{m}\left(A_{2} \circ B_{2}\right)\right\|_{p}\right\} \text { for } n=2 m+1 .
\end{aligned}
$$

Proof. For $n=2 m$, by equation (9) and part (1) in Lemma 2, we have

$$
\begin{aligned}
\rho(A \circ B) & =\rho\left(\left(A_{1} \circ B_{1}\right)+J_{m}\left(A_{2} \circ B_{2}\right)\right) \\
& \leq\left\|A_{1} \circ B_{1}+J_{m}\left(A_{2} \circ B_{2}\right)\right\|_{p} \\
& \leq\left\|A_{1} \circ B_{1}\right\|_{p}+\left\|J_{m}\left(A_{2} \circ B_{2}\right)\right\|_{p} \\
& \leq\left\|A_{1} \circ B_{1}\right\|_{p}+\left\|J_{m}\right\|_{p}\left\|A_{2} \circ B_{2}\right\|_{p} .
\end{aligned}
$$

For any nonnegative matrices, we have $\|A \circ B\| \leq \rho\left(A^{T} B\right)$ by [6], then

$$
\rho(A \circ B) \leq \rho\left(A_{1}^{T} B_{1}\right)+\left\|J_{m}\right\|_{p} \rho\left(A_{2}^{T} B_{2}\right) .
$$

Since $\left\|J_{m}\right\|_{p}=m^{1 / p}$, we get the result. The second inequality follows directly.

Lemma 4. Let $X, Y, Z, W \in \mathbb{C}^{n \times n}$. Then, for $2 \leq p<\infty$,

$$
\left\|\left[\begin{array}{ll}
X & Y \\
W & Z
\end{array}\right]\right\|_{p} \leq 2^{1 / p}\left(\frac{p-1}{2} \operatorname{Tr}\left(\alpha^{2}\right)+\frac{2-p}{4}(\operatorname{Tr}(\alpha))^{2}\right)^{1 / 2}
$$

where

$$
\alpha=\left[\begin{array}{cc}
\|X\|_{p} & \left(\frac{1}{2}\left(\|Y\|_{p}^{p}+\|W\|_{p}^{p}\right)\right)^{1 / p} \\
\left(\frac{1}{2}\left(\|Y\|_{p}^{p}+\|W\|_{p}^{p}\right)\right)^{1 / 2} & \|Z\|_{p}
\end{array}\right]
$$

as in [7].

Now we give an upper bound for the spectral radius of Hadamard product of two centrosymmetric matrices in terms of $p$-norms.

Theorem 2. Let $A$ and $B$ be two centrosymmetric matrices as in (7) or(8), and let $2 \leq p<\infty$. Then for $n=2 m$ and $n=2 m+1$, respectively, we have

$$
\begin{aligned}
\rho(A \circ B) \leq & 2^{1 / p}(p-1)\left(1+m^{2}\right)\left(\|P\|_{p}^{2}+\|S\|_{p}^{2}\right)^{1 / 2} \\
\rho(A \circ B) & \leq\left\|P-J_{m} S\right\|_{p}+2^{1 / p}\left(\frac{p-1}{2}\left(\alpha^{2} \beta^{2}+2 M^{2}+\left\|P+J_{m} S\right\|_{p}^{2}\right)\right. \\
& \left.+\frac{2-p}{4}\left(\alpha \beta+\left\|P+J_{m} S\right\|_{p}\right)^{2}\right)^{1 / 2}
\end{aligned}
$$

where $P=A_{1} \circ B_{1}, S=A_{2} \circ B_{2}$, and

$$
M=\left\{\frac{1}{2}\left(\left\|\sqrt{2} J_{m}\left(a_{2} \circ b_{2}\right)\right\|_{p}^{p}+\left\|\sqrt{2}\left(a_{1}^{T} \circ b_{1}^{T}\right)\right\|_{p}^{p}\right)\right\}^{1 / p} .
$$


Proof. If $n=2 m$, we define a symmetric matrix such that

$$
\begin{aligned}
\alpha & =\left[\begin{array}{cc}
\left\|A_{1} \circ B_{1}\right\|_{p} & \left(\frac{1}{2}\left(\left\|J_{m}\left(A_{2} \circ B_{2}\right) J_{m}\right\|_{p}^{p}+\left\|A_{2} \circ B_{2}\right\|_{p}^{p}\right)\right)^{1 / p} \\
\left(\frac{1}{2}\left(\left\|J_{m}\left(A_{2} \circ B_{2}\right) J_{m}\right\|_{p}^{p}+\left\|A_{2} \circ B_{2}\right\|_{p}^{p}\right)\right)^{1 / p} & \left\|J_{m}\left(A_{1} \circ B_{1}\right) J_{m}\right\|_{p}
\end{array}\right] \\
& =\left[\begin{array}{cc}
\|P\|_{p} \\
\left(\frac{1}{2}\left(\left\|J_{m} S J_{m}\right\|_{p}^{p}+\|S\|_{p}^{p}\right)\right)^{1 / p}
\end{array} \begin{array}{c}
\left(\frac{1}{2}\left(\left\|J_{m} S J_{m}\right\|_{p}^{p}+\|S\|_{p}^{p}\right)\right)^{1 / p} \\
\left\|J_{m} P J_{m}\right\|_{p}
\end{array}\right]
\end{aligned}
$$

Note that since $\left\|J_{m}\right\|_{p}=\left(\operatorname{Tr}\left(J_{m}^{*} J_{m}\right)^{p / 2}\right)^{1 / p}=\left(\operatorname{Tr}\left(I_{m}\right)\right)^{1 / p}=m^{1 / p}$, we have

$$
\begin{aligned}
\left(\frac{1}{2}\left(\left\|J_{m} S J_{m}\right\|_{p}^{p}+\|S\|_{p}^{p}\right)\right)^{2 / p} & \leq 2^{-2 / p}\left(\left\|J_{m}\right\|_{p}^{p}\|S\|_{p}^{p}\left\|J_{m}\right\|_{p}^{p}+\|S\|_{p}^{p}\right)^{2 / p} \\
& =2^{-2 / p}\left(\left(m^{2}+1\right)\|S\|_{p}^{p}\right)^{2 / p} \\
& =2^{-2 / p}\left(m^{2}+1\right)^{2 / p}\|S\|_{p}^{2}
\end{aligned}
$$

Then we get

$$
\begin{aligned}
(\operatorname{Tr}(\alpha))^{2} & =\left(\|P\|_{p}+\left\|J_{m} P J_{m}\right\|_{p}\right)^{2} \\
& \leq\left(\|P\|_{p}+\left\|J_{m}\right\|_{p}\|P\|_{p}\left\|J_{m}\right\|_{p}\right)^{2} \\
& \leq\left(\|P\|_{p}+m^{2 / p}\|P\|_{p}\right)^{2}=\left(1+m^{2 / p}\right)^{2}\|P\|_{p}^{2}
\end{aligned}
$$

and by equation (10), we obtain

$$
\begin{aligned}
\operatorname{Tr}(\alpha)^{2} & =\|P\|_{p}^{2}+\left\|J_{m} P J_{m}\right\|_{p}^{2}+2\left(\frac{1}{2}\left(\left\|J_{m} S J_{m}\right\|_{p}^{p}+\|S\|_{p}^{p}\right)\right)^{2 / p} \\
& \leq\|P\|_{p}^{2}+m^{4 / p}\|P\|_{p}^{2}+2^{1-2 / p}\left(m^{2}+1\right)^{2 / p}\|S\|_{p}^{2} \\
& =\left(m^{4 / p}+1\right)\|P\|_{p}^{2}+2^{1-2 / p}\left(m^{2}+1\right)^{2 / p}\|S\|_{p}^{2}
\end{aligned}
$$

Thus, since $\|A \circ B\|_{p}=\left\|Q_{1}(A \circ B) Q_{1}\right\|_{p}$ and $Q_{1}(A \circ B) Q_{1}$ is as in (7), by Lemma 4 , for $p \geq 2$ we have

$$
\begin{aligned}
\rho(A \circ B) & \leq\|A \circ B\|_{p} \\
& \leq 2^{1 / p}\left(\frac{p-1}{2}\left(m^{4 / p}+1\right)\|P\|_{p}^{2}+(p-1) 2^{-2 / p}\left(m^{2}+1\right)^{2 / p}\|S\|_{p}^{2}+\frac{2-p}{4}\left(1+m^{2 / p}\right)^{2}\|P\|_{p}^{2}\right)^{1 / 2} \\
& =2^{1 / p}\left(\frac{p}{4}\left(m^{4 / p}+1\right)\|P\|_{p}^{2}+m^{2 / p}\left(1-\frac{p}{2}\right)\|P\|_{p}^{2}+(p-1) 2^{-2 / p}\left(m^{2}+1\right)^{2 / p}\|S\|_{p}^{2}\right)^{1 / 2} \\
& =2^{1 / p}\left(\frac{p}{4}\left(m^{4 / p}-\frac{2(p-2)}{p} m^{2 / p}+1\right)\|P\|_{p}^{2}+(p-1) 2^{-2 / p}\left(m^{2}+1\right)^{2 / p}\|S\|_{p}^{2}\right)^{1 / 2} \\
& \leq 2^{1 / p}\left((p-1)\left(m^{2 / p}+1\right)^{2}\|P\|_{p}^{2}+(p-1) 2^{-2 / p}\left(m^{2}+1\right)^{2 / p}\|S\|_{p}^{2}\right)^{1 / 2} \\
& \leq 2^{1 / p}\left((p-1)\left(m^{2}+1\right)^{2}\|P\|_{p}^{2}+(p-1) 2^{-2 / p}\left(m^{2}+1\right)^{2}\|S\|_{p}^{2}\right)^{1 / 2} \\
& \leq 2^{1 / p}(p-1)\left(m^{2}+1\right)\left(\|P\|_{p}^{2}+\|S\|_{p}^{2}\right)^{1 / 2} \cdot
\end{aligned}
$$


Inequalities follow from the fact that $m^{4 / p}-\frac{2(p-2)}{p} m^{2 / p}+1 \leq\left(m^{2 / p}+1\right)^{2}$ and $2^{-2 / p}<1$ for $p \geq 2$.

If $n=2 m+1$, note that

$$
\rho(A \circ B) \leq\|A \circ B\|_{p}=\left\|Q_{2}^{T}(A \circ B) Q_{2}\right\|_{p}=\left\|A_{1} \circ B_{1}-J_{m}\left(A_{2} \circ B_{2}\right)\right\|_{p}+\|K\|_{p}
$$

where $K=\left[\begin{array}{cc}\alpha \beta & \sqrt{2}\left(a_{1}^{T} \circ b_{1}^{T}\right) \\ \sqrt{2} J_{m}\left(a_{2} \circ b_{2}\right) & \left(A_{1} \circ B_{1}\right)+J_{m}\left(A_{2} \circ B_{2}\right)\end{array}\right]$. Then define a symmetric matrix $\tilde{K}$ such that

$$
\begin{aligned}
\tilde{K} & =\left[\begin{array}{c}
\alpha \beta \\
\left(\frac{1}{2}\left(\left\|\sqrt{2} J_{m}\left(a_{2} \circ b_{2}\right)\right\|_{p}^{p}+\left\|\sqrt{2}\left(a_{1}^{T} \circ b_{1}^{T}\right)\right\|_{p}^{p}\right)\right)^{1 / p}
\end{array}\right. \\
& =\left[\begin{array}{l}
\alpha \beta \\
M\left\|P+J_{m} S\right\|_{p}
\end{array}\right] .
\end{aligned}
$$

Then $\operatorname{Tr}(\tilde{K})=\alpha \beta+\left\|P+J_{m} S\right\|_{p}$ and $\operatorname{Tr}\left(\tilde{K}^{2}\right)=\alpha^{2} \beta^{2}+2 M^{2}+\left\|P+J_{m} S\right\|_{p}^{2}$. By Lemma 4, we have

$$
\|K\|_{p} \leq 2^{1 / p}\left[\frac{1}{2}\left(\alpha^{2} \beta^{2}+2 M^{2}+\left\|P+J_{m} S\right\|_{p}^{2}\right)+\frac{2-p}{4}\left(\alpha \beta+\left\|P+J_{m} S\right\|_{p}\right)^{2}\right]^{1 / 2}
$$

Thus, combining equations (11) and (12), we obtain the result.

\section{Conclusion}

The algebraic relationships between the Kronecker and Hadamard product of centrosymmetric matrices have been examined in this work. Specifically, the upper bound for the spectral radius of Hadamard product of two centrosymmetric matrices have been obtained analytically.

Furthermore, the alternative proof of the statement which has two different dimensions of the Hadamard product of two centrosymmetric matrices have been pointed out. Finally, the upper bound for the spectral radius of Hadamard product of two centrosymmetric matrices in terms of p-norms of submatrices were given.

\section{Competing interests}

The authors declare that they have no competing interests.

\section{Authors' contributions}

All authors have contributed to all parts of the article. All authors read and approved the final manuscript.

\section{References}

[1] Koenraad MR Audenaert. Spectral radius of hadamard product versus conventional product for non-negative matrices. Linear Algebra and its Applications, 432(1):366-368, 2010. 
[2] John W Brewer. Kronecker products and matrix calculus in system theory. Circuits and Systems, IEEE Transactions on, 25(9):772781, 1978.

[3] Wen Chen, W Wang, and Tingxiu Zhong. The structure of weighting coefficient matrices of harmonic differential quadrature and its applications. Numerical Methods in Engineering, 12:455-459, 1996.

[4] Lokesh Datta and Salvatore D Morgera. On the reducibility of centrosymmetric matrices applications in engineering problems. Circuits, Systems and Signal Processing, 8(1):71-96, 1989.

[5] Roger A Horn and Charles R Johnson. Matrix analysis. Cambridge University Press, New York, 1985.

[6] Zejun Huang. On the spectral radius and the spectral norm of hadamard products of nonnegative matrices. Linear Algebra and its Applications, 434(2):457-462, 2011.

[7] Christopher King. Inequalities for trace norms of $2 \times 2$ block matrices. Communications in mathematical physics, 242(3):531-545, 2003.

[8] Hongyi Li, Di Zhao, Fei Dai, and Donglin Su. On the spectral radius of a nonnegative centrosymmetric matrices. Applied Mathematics and Computation, 218(9):4962-4966, 2012.

[9] Zhong-Yun Liu. Some properties of centrosymmetric matrices. Applied mathematics and computation, 141(2):297-306, 2003.

[10] Bertram Mond and Josip Pečarić. On inequalities involving the hadamard product of matrices. ELA. The Electronic Journal of Linear Algebra, 6:56-61, 2000.

[11] Di Zhao, Hongyi Li, and Zhiguo Gong. Trace inequalities for positive semidefinite matrices with centrosymmetric structure. Journal of Inequalities and Applications, 2012(1):1-6, 2012. 